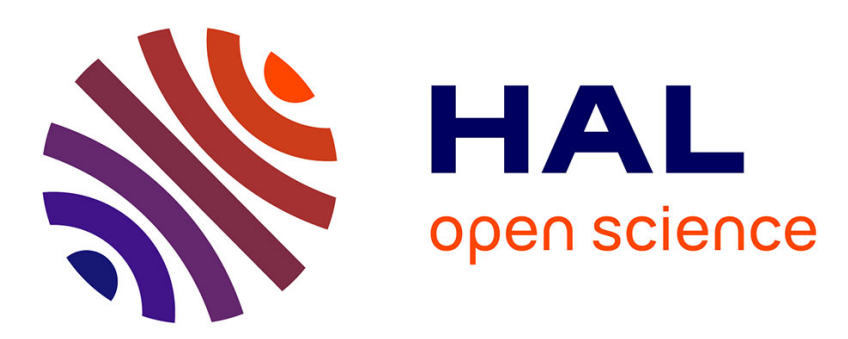

\title{
Comparison of 3-RPS and 3-SPR Parallel Manipulators based on their Maximum Inscribed Singularity-free Circle
}

\author{
Abhilash Nayak, Latifah Nurahmi, Philippe Wenger, Stéphane Caro
}

\section{To cite this version:}

Abhilash Nayak, Latifah Nurahmi, Philippe Wenger, Stéphane Caro. Comparison of 3-RPS and 3SPR Parallel Manipulators based on their Maximum Inscribed Singularity-free Circle. New Trends in Mechanism and Machine Science Theory and Industrial Applications, 43, pp.121-130, 2017, Mechanisms and Machine Science, 10.1007/978-3-319-44156-6_13 . hal-01758006

\section{HAL Id: hal-01758006 https://hal.science/hal-01758006}

Submitted on 4 Apr 2018

HAL is a multi-disciplinary open access archive for the deposit and dissemination of scientific research documents, whether they are published or not. The documents may come from teaching and research institutions in France or abroad, or from public or private research centers.
L'archive ouverte pluridisciplinaire HAL, est destinée au dépôt et à la diffusion de documents scientifiques de niveau recherche, publiés ou non, émanant des établissements d'enseignement et de recherche français ou étrangers, des laboratoires publics ou privés. 


\title{
Comparison of 3-RPS and 3-SPR Parallel Manipulators based on their Maximum Inscribed Singularity-free Circle
}

\author{
Abhilash Nayak ${ }^{1}$, Latifah Nurahmi ${ }^{2}$, Philippe Wenger ${ }^{3}$ and Stéphane Caro ${ }^{3}$ \\ ${ }^{1}$ École Centrale de Nantes, IRCCyN, France, e-mail: \\ Abhilash.Nayak@irccyn.ec-nantes.fr \\ ${ }^{2}$ Mechanical Engineering, Institut Teknologi Sepuluh Nopember, Surabaya, \\ Indonesia,e-mail: latifah.nurahmi@gmail.com \\ ${ }^{3}$ CNRS, IRCCyN, France, e-mail: \{Philippe.Wenger, \\ Stephane.Caro\}@irccyn.ec-nantes.fr
}

\begin{abstract}
This paper deals with the comaprison of 3-RPS and 3-SPR parallel manipulators based on their operation modes and singularity-free workspace. The operation modes of the 3-SPR manipulator are identified by using Algebraic Geometry. Those operation modes amount to the operation modes of the 3-RPS parallel manipulator, which has already been studied in the literature [1]. Then, the parallel singularities of the 3-SPR and 3-RPS parallel manipulators are analyzed in order to trace their singularity loci in the orientation workspace. An index, named Maximum Inscribed Circle Radius (MICR), is defined to compare the two manipulators under study. It is based on their maximum singularity-free workspace and the ratio between their circum-radius of the movingplatform to that of the base.
\end{abstract}

Key words: 3 -RPS parallel manipulator, $3-\mathrm{S} \underline{P R}$ parallel manipulator, operation modes, singularity analysis, maximum inscribed circle radius

\section{Introduction}

Zero torsion parallel mechanisms have proved to be interesting and versatile. In this regard, the three degree of freedom lower mobility 3-RPS parallel manipulator (PM) has many practical applications and has been analyzed by many researchers $[1,2]$. Interchanging the free moving platform and the fixed base in 3-RPS manipulator results in the 3-SPR manipulator as shown in figure 1, retaining three degrees of freedom.

The study of 3-SPR is limited in the literature. An optimization algorithm was used in [3] to compute the forward and inverse kinematics of 3-SPR manipulator. After the workspace generation it is proved that the 3-SPR has a bigger working space volume compared to the 3-RPS manipulator. The orthogonality of rotation matrices is exploited in [4] to perform the forward and inverse kinematics along with the simulations of 3-SPR mechanism. Control of a hydraulic actuated 3-SPR $\mathrm{PM}$ is demonstrated in [5] with an interesting application on adaptive shell structure.

This paper focuses on the comparison of kinematics and singularities of the 3 RPS and 3-SPR parallel manipulators and is organized as follows: initially, the de- 
sign of 3-SPR PM is detailed and the design of the 3-RPS PM is recalled. The second section describes the derivation of the constraint equations of the 3-SPR manipulator based on the algebraic geometry approach $[1,6]$. The primary decomposition is computed over these constraint equations and it shows that the 3-SPR has identical operation modes as the 3-RPS PM. Moreover, the actuation and constraint singularities are described with singularity loci plots in the orientation workspace. Finally, an index called the singularity-free maximum inscribed circle radius is introduced to compare the maximum singularity free regions of 3-RPS and 3-SPR manipulators from their home position. In [7], maximum tilt angles for any azimuth for 3-RPS PM are plotted for different ratios of platform to base circumradii. However, these plots correspond to only one operation mode since the notion of operation modes was not considered in this paper. That being the case, this paper offers a complete singularity analysis in terms of MICR for both the manipulators. These plots are useful in the design choice of a manipulator based on their platform to base circumradii ratios and their operation modes.

\section{Manipulator architectures}

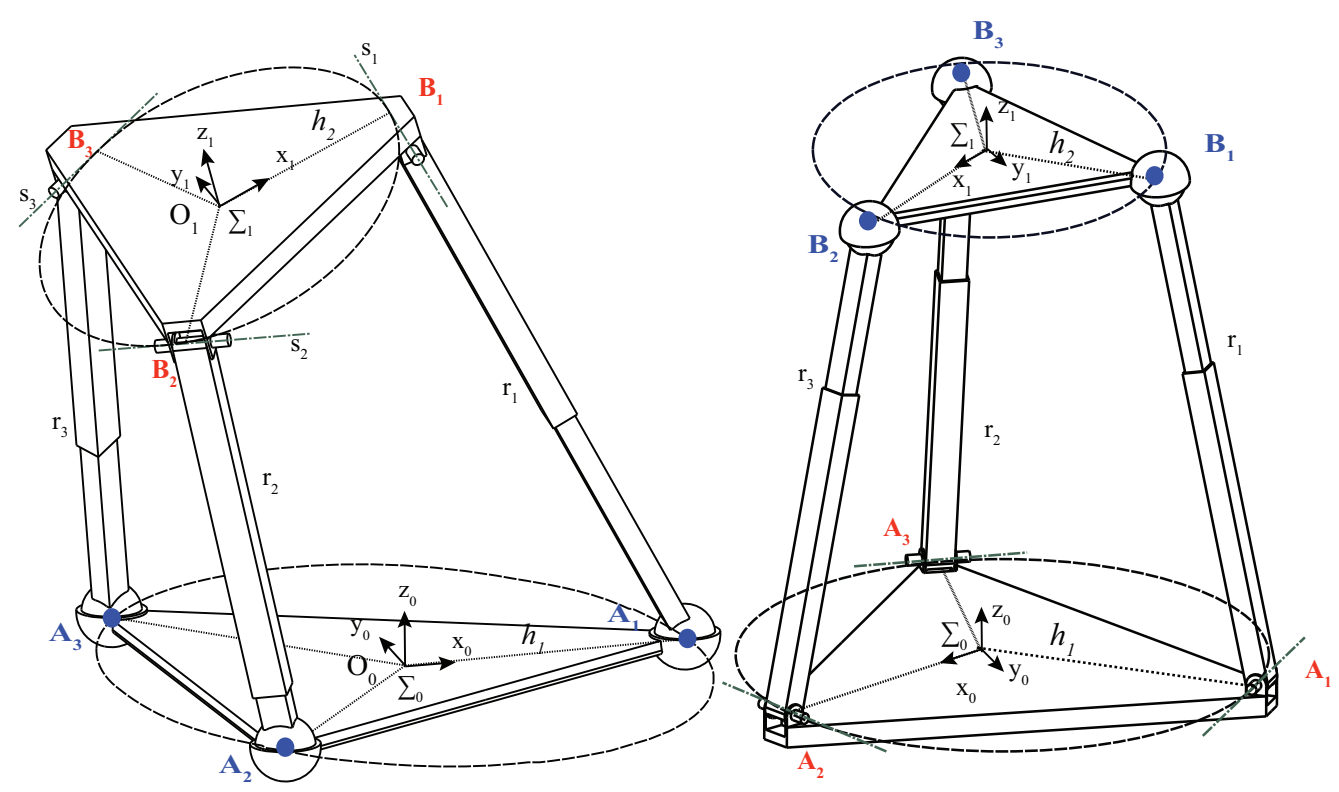

Fig. 13 -SPR parallel manipulator

Fig. 2 3-R $\underline{\text { PS }}$ parallel manipulator

Figure 1 shows a general pose of the 3-SPR parallel manipulator with 3 identical legs each comprising of a spherical, prismatic and revolute joints. The triangular base and the platform of the manipulator are equilateral. 
$\Sigma_{0}$ is the fixed co-ordinate frame attached to the base with the origin $O_{0}$ coinciding with the circum-centre of the triangular base. The centres of the spherical joints, namely $A_{1}, A_{2}$ and $A_{3}$ bound the triangular base. $x_{0}$-axis of $\Sigma_{0}$ is considered along $O_{0} A_{1}$ which makes the $y_{0}$-axis parallel to $A_{2} A_{3}$ and the $z_{0}$-axis normal to the triangular base plane. $h_{2}$ is the circum-radius of the triangular base.

The moving platform is bounded by three points $B_{1}, B_{2}$ and $B_{3}$ that lie on the revolute joint axes $\mathbf{s}_{1}, \mathbf{s}_{2}$ and $\mathbf{s}_{3}$. Moving co-ordinate frame $\Sigma_{1}$ is attached to the moving platform whose $x_{1}$-axis points from the origin $O_{1}$ to $B_{1}, y_{1}$-axis being orthogonal to the line segment $B_{2} B_{3}$ and the $z_{1}$-axis normal to the triangular platform. Circum-radius of this triangle with $B_{i}(i=1,2,3)$ as vertices is defined as $h_{2}$.

The prismatic joint of the $i$-th $(i=1,2,3)$ leg is always perpendicular to the respective revolute joint axis in each leg. Hence the prevailing orthogonality of $A_{i} B_{i}$ to $\mathbf{s}_{i}(i=1,2,3)$ no matter the motion of the platform is a constraint of the manipulator. The distance between the points $A_{i}$ and $B_{i}(i=1,2,3)$ is defined by the prismatic joint variables $r_{i}$.

The architecture of the 3-SPR PM is similar to that of the 3-RPS PM except that the order of the joints in each leg is reversed. The architecture of 3-RPS is recalled in figure 2 where the revolute joints are attached to the fixed triangular base with circum-radius $h_{1}$ while the spherical joints are attached to the moving platform with circum-radius $h_{2}$.

\section{Constraint equations of the 3-SPR parallel manipulator}

The homogeneous coordinates of $A_{i}$ and $B_{i}$ in the frames $\Sigma_{0}$ and $\Sigma_{1}$ respectively are expressed as follows:

$$
\begin{array}{lll}
\mathbf{r}_{A_{1}}^{0}=\left[1, h_{1}, 0,0\right]^{T}, & \mathbf{r}_{A_{2}}^{0}=\left[1,-\frac{1}{2} h_{1},-\frac{1}{2 \sqrt{3}} h_{1}, 0\right]^{T}, & \mathbf{r}_{A_{3}}^{0}=\left[1,-\frac{1}{2} h_{1}, \frac{1}{2 \sqrt{3}} h_{1}, 0\right]^{T} \\
\mathbf{r}_{B_{1}}^{1}=\left[1, h_{2}, 0,0\right]^{T}, & \mathbf{r}_{B_{2}}^{1}=\left[1,-\frac{1}{2} h_{2},-\frac{1}{2 \sqrt{3}} h_{2}, 0\right]^{T}, & \mathbf{r}_{B_{3}}^{1}=\left[1,-\frac{1}{2} h_{2}, \frac{1}{2 \sqrt{3}} h_{2}, 0\right]^{T}
\end{array}
$$

To express the coordinates of $B_{i}$ in the frame $\Sigma_{0}$, a coordinate transformation matrix must be used. In this context, the Study parametrization of a spatial Euclidean transformation matrix $\mathbf{M} \in S E(3)$ is utilized and is represented as:

$$
\mathbf{M}=\left[\begin{array}{cc}
x_{0}^{2}+x_{1}{ }^{2}+x_{2}{ }^{2}+x_{3}{ }^{2} & \mathbf{0}_{3 \times 1}^{T} \\
\mathbf{M}_{T} & \mathbf{M}_{R}
\end{array}\right], \quad \mathbf{M}_{T}=\left[\begin{array}{c}
-2 x_{0} y_{1}+2 x_{1} y_{0}-2 x_{2} y_{3}+2 x_{3} y_{2} \\
-2 x_{0} y_{2}+2 x_{1} y_{3}+2 x_{2} y_{0}-2 x_{3} y_{1} \\
-2 x_{0} y_{3}-2 x_{1} y_{2}+2 x_{2} y_{1}+2 x_{3} y_{0}
\end{array}\right]
$$




$$
\mathbf{M}_{R}=\left[\begin{array}{ccc}
x_{0}^{2}+x_{1}^{2}-x_{2}^{2}-x_{3}^{2} & -2 x_{0} x_{3}+2 x_{1} x_{2} & 2 x_{0} x_{2}+2 x_{1} x_{3} \\
2 x_{0} x_{3}+2 x_{1} x_{2} & x_{0}^{2}-x_{1}^{2}+x_{2}^{2}-x_{3}^{2} & -2 x_{0} x_{1}+2 x_{3} x_{2} \\
-2 x_{0} x_{2}+2 x_{1} x_{3} & 2 x_{0} x_{1}+2 x_{3} x_{2} & x_{0}^{2}-x_{1}^{2}-x_{2}^{2}+x_{3}^{2}
\end{array}\right]
$$

where $\mathbf{M}_{T}$ and $\mathbf{M}_{R}$ represent the translational and rotational parts of the transformation matrix $\mathbf{M}$ respectively. The parameters $x_{i}, y_{i}, i \in\{0, \ldots, 3\}$ are called the Study parameters. Matrix $\mathbf{M}$ maps every displacement $S E(3)$ to a point in a 7dimensional projective space $\mathbb{P}^{7}$ and this mapping is known as Study's kinematic mapping. An Euclidean transformation will be represented by a point $\mathrm{P} \in \mathbb{P}^{7}$ if and only if the following equation and inequality are satisfied:

$$
\begin{aligned}
x_{0} y_{0}+x_{1} y_{1}+x_{2} y_{2}+x_{3} y_{3} & =0 \\
x_{0}{ }^{2}+x_{1}{ }^{2}+x_{2}{ }^{2}+x_{3}{ }^{2} & \neq 0
\end{aligned}
$$

All the points that satisfy equation (3) belong to the 6-dimensional Study quadric. The points that do not satisfy the inequality (4) lie in the exceptional generator $x_{0}=$ $x_{1}=x_{2}=x_{3}=0$. To derive the constraint equations, we can express the direction of the vectors $\mathbf{s}_{1}, \mathbf{s}_{2}$ and $\mathbf{s}_{3}$ in homogeneous coordinates in frame $\Sigma_{1}$ as:

$$
\mathbf{s}_{1}^{1}=[1,0,-1,0]^{T}, \quad \mathbf{s}_{2}^{1}=\left[1,-\frac{1}{2 \sqrt{3}}, \frac{1}{2}, 0\right]^{T}, \quad \mathbf{s}_{3}^{1}=\left[1, \frac{1}{2 \sqrt{3}}, \frac{1}{2}, 0\right]^{T}
$$

In the fixed coordinate frame $\Sigma_{0}, B_{i}$ and $\mathbf{s}_{i}$ can be expressed using the transformation matrix $\mathbf{M}$ :

$$
\mathbf{r}_{B_{i}}^{0}=\mathbf{M} \mathbf{r}_{B_{i}}^{1} ; \quad \mathbf{s}_{i}^{0}=\mathbf{M} \mathbf{s}_{i}^{1} \quad i=1,2,3
$$

As it is clear from the manipulator architecture, the vector along $A_{i} B_{i}$, namely $\mathbf{r}_{B_{i}}^{0}-\mathbf{r}_{A_{i}}^{0}$ is orthogonal to the axis $\mathbf{s}_{i}$ of the $i$-th revolute joint which after simplification yields the following three equations:

$$
\left(\mathbf{r}_{B_{i}}^{0}-\mathbf{r}_{A_{i}}^{0}\right)^{T} \mathbf{s}_{i}=0 \Longrightarrow\left\{\begin{array}{l}
g_{1}:=x_{0} x_{3}=0 \\
g_{2}:=h_{1} x_{1}^{2}-h_{1} x_{2}^{2}-2 x_{0} y_{1}+2 x_{1} y_{0}+2 x_{2}-2 x_{3} y_{2}=0 \\
g_{3}:=2 h_{1} x_{0} x_{3}+h_{1} x_{1} x_{2}+x_{0} y_{2}+x_{1} y_{3}-x_{2} y_{0}-x_{3} y_{1}=0
\end{array}\right.
$$

The actuation of prismatic joints leads to three additional constraint equations. The Euclidean distance between $A_{i}$ and $B_{i}$ must be equal to $r_{i}$ for the $i$-th leg of the manipulator. As a result, $\left\|A_{i} B_{i}\right\|^{2}=r_{i}^{2}$ leads to three additional equations $g_{4}=g_{5}=g_{6}=0$, which are quite lengthy and are not displayed in this paper due to space limitation.

Two other equations are considered such that the solution represents a transformation in $S E$ (3). The study-equation $g_{7}=0$ in Equation (3) constrains the solutions to lie on the Study quadric. $g_{8}=0$ is the normalization equation respecting the inequality (4). Solving these eight constraint equations provides the direct kinematic solutions for the 3-SPR parallel manipulator. 


$$
g_{7}:=x_{0} y_{0}+x_{1} y_{1}+x_{2} y_{2}+x_{3} y_{3}=0 \quad ; \quad g_{8}:=x_{0}^{2}+x_{1}^{2}+x_{2}^{2}+x_{3}^{2}-1=0
$$

\section{Operation modes}

Algebraic geometry offers an organized and an effective methodology to deal with the eight constraint equations. A polynomial ideal consisting of equations $g_{i}(i=$ $1, \ldots, 8)$ is defined with variables $\left\{x_{0}, x_{1}, x_{2}, x_{3}, y_{0}, y_{1}, y_{2}, y_{3}\right\}$ over the coefficient ring $\mathbb{C}\left[h_{1}, h_{2}, r_{1}, r_{2}, r_{3}\right]$ as follows:

$$
\mathcal{I}=<g_{1}, g_{2}, g_{3}, g_{4}, g_{5}, g_{6}, g_{7}, g_{8}>
$$

The vanishing set or the variety $V(\mathcal{I})$ of this ideal $\mathcal{I}$ consists of the solution to direct kinematics as points in $\mathbb{P}^{7}$. However, in this context, only the number of operation modes are of concern irrespective of the joint variable values. Hence, the sub-ideal independent of the prismatic joint length, $r_{i}$ is considered:

$$
\mathcal{J}=<g_{1}, g_{2}, g_{3}, g_{7}>
$$

The primary decomposition of ideal $\mathcal{J}$ is calculated to obtain three simpler ideals $\mathcal{J}_{i}(i=1,2,3)$. The intersection of the resulting primary ideals returns the ideal $\mathcal{J}$. From a geometrical viewpoint, the variety $V(\mathcal{J})$ can be written as the union of the varieties of the primary ideals $V\left(\mathcal{J}_{i}\right), i=1,2,3$ [8].

$$
\mathcal{J}=\bigcap_{i=1}^{3} \mathcal{J}_{i} \quad \text { or } \quad V(\mathcal{J})=\bigcup_{i=1}^{3} V\left(\mathcal{J}_{i}\right)
$$

Among the three primary ideals obtained as a result of primary decomposition, it is important to note that $\mathcal{J}_{1}$ and $\mathcal{J}_{2}$ contain $x_{0}$ and $x_{3}$ as their first elements, respectively. The third ideal, $\mathcal{J}_{3}$ is obtained as $\mathcal{J}_{3}=<x_{0}, x_{1}, x_{2}, x_{3}>$ and is discarded as the variety $V\left(\mathcal{J}_{3} \cup g_{8}\right)$ is null over the field of interest $\mathbb{C}$. As a result, the 3-SPR PM has two operation modes, represented by $x_{0}=0$ and $x_{3}=0$. In fact, $g_{1}=0$ in Equation (7) shows the presence of these two operation modes. It is noteworthy that the 3-RPS PM also has two operation modes as described in [2].

The analysis is completed by adding the remaining constraint equations to the primary ideals $\mathcal{J}_{1}$ and $\mathcal{J}_{2}$. Accordingly, two ideals $\mathcal{K}_{1}$ and $\mathcal{K}_{2}$ are obtained. As a consequence, the ideals $\mathcal{K}_{i}$ correspond to the two operation modes and can be studied separately.

$$
\mathcal{K}_{i}=\mathcal{J}_{i} \cup<g_{4}, g_{5}, g_{6}, g_{8}>\quad i=1,2
$$

The system of equations in the ideals $\mathcal{K}_{1}$ and $\mathcal{K}_{2}$ can be solved for a particular set of joint variables to obtain the Study parameters and hence the pose of the manipulator. These Study parameters can be substituted back in equation (2) to obtain the transformation matrix M. According to the theorem of Chasles this matrix now rep- 
resents a discrete screw motion from the identity position (when the fixed frame $\Sigma_{0}$ and the moving frame $\Sigma_{1}$ coincide) to the moving-platform pose. The displacement about the corresponding discrete screw axis (DSA) defines the pose of the moving platform.

\subsection{Ideal $\mathcal{K}_{1}:$ Operation mode $1: x_{0}=0$}

For operation mode 1, the moving platform is always found to be displaced about a DSA by 180 degrees [9]. Substituting $x_{0}=0$ and solving for $y_{0}, y_{1}, y_{3}$ from the ideal $\mathcal{K}_{1}$ shows that the translational motions can be parametrized by $y_{2}$ and the rotational motions by $x_{1}, x_{2}$ and $x_{3}[10]$.

\subsection{Ideal $\mathcal{K}_{2}:$ Operation mode $2: x_{3}=0$}

For operation mode 2, the moving platform is displaced about a DSA with a rotation angle $\alpha$ calculated from $\cos \left(\frac{\alpha}{2}\right)=x_{0}$. It is interesting to note that the DSA in this case is always parallel to the xy-plane [9]. Substituting $x_{3}=0$ and solving for $y_{0}, y_{2}, y_{3}$ from the ideal $\mathcal{K}_{2}$ shows that the translational motions can be parametrized by $y_{1}$ and the rotational motions by $x_{0}, x_{1}$ and $x_{2}$ [10].

\section{Singularity analysis}

The Jacobian of the 3-SPR manipulator in this context is defined as $\mathbf{J}_{i}$ and the manipulator reaches a singular position when its determinant vanishes.:

$$
\mathbf{J}_{i}=\left(\frac{\partial g_{j}}{\partial x_{k}}, \frac{\partial g_{j}}{\partial y_{k}}\right) \text { where } i=1,2 ; \quad j=1, \ldots, 8 ; \quad k=0, \ldots, 3
$$

\subsection{Actuation and constraint singularities}

Computing the determinant $S_{i}: \operatorname{det}\left(\mathbf{J}_{i}\right)$ results in a hyper-variety of degree 8 in both the operation modes:

$S_{1}: x_{3} \cdot p^{7}\left(x_{1}, x_{2}, x_{3}, y_{0}, y_{1}, y_{2}, y_{3}\right)=0 \quad$ and $\quad S_{2}: x_{0} \cdot p^{7}\left(x_{0}, x_{1}, x_{2}, y_{0}, y_{1}, y_{2}, y_{3}\right)=0$ 
The 7 degree polynomials describe the actuation singularities when the prismatic joints are actuated and that exist within each operation mode whereas $x_{0}=x_{3}=0$ describes the constraint singularity that exhibits the transition between $\mathcal{K}_{1}$ and $\mathcal{K}_{2}$.

\subsection{Singularity Loci}

The actuation singularities can be expressed in the orientation workspace by parametrizing the orientation of the platform in terms of Euler angles. In particular, the Study parameters can be expressed in terms of the Euler angles azimuth $(\phi)$, tilt $(\theta)$ and torsion $(\psi)[?]$ :

$$
\begin{aligned}
& x_{0}=\cos \left(\frac{\theta}{2}\right) \cos \left(\frac{\phi}{2}+\frac{\psi}{2}\right) \quad x_{1}=\sin \left(\frac{\theta}{2}\right) \cos \left(\frac{\phi}{2}-\frac{\psi}{2}\right) \\
& x_{2}=\sin \left(\frac{\theta}{2}\right) \sin \left(\frac{\phi}{2}-\frac{\psi}{2}\right) \quad x_{3}=\cos \left(\frac{\theta}{2}\right) \sin \left(\frac{\phi}{2}+\frac{\psi}{2}\right)
\end{aligned}
$$

Since $\mathcal{K}_{1}$ and $\mathcal{K}_{2}$ are characterized by $x_{0}=0$ and $x_{3}=0$, substituting them in equation (15) makes the torsion angle $(\psi)$ null, verifying the fact that, like its 3RPS counterpart, the 3-SPR parallel manipulator is a zero-torsion manipulator. Accordingly, the $x_{i}$ parameters can be written in terms of $\operatorname{tilt}(\theta)$ and azimuth $(\phi)$ only. The following method is used to calculate the determinant of $\mathbf{J}_{i}$ in terms

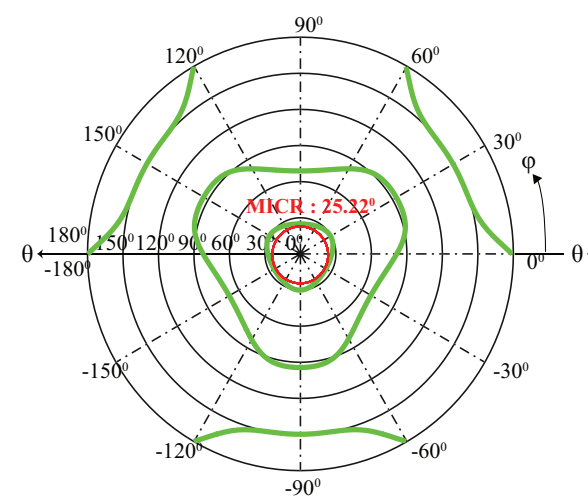

(a)

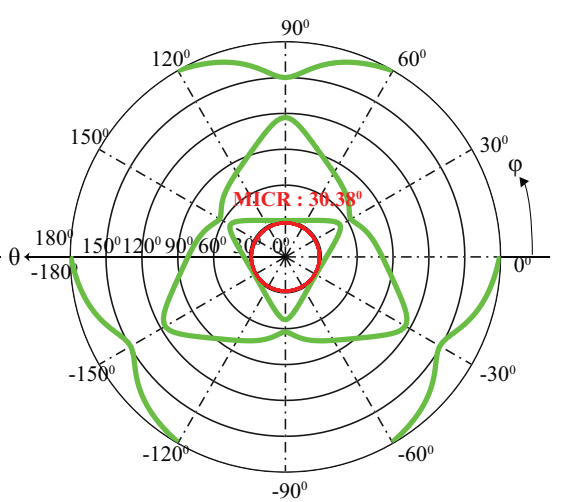

(b)

Fig. 3 3-SPR singularity loci and the maximum inscribed singularity-free circle (a) Operation mode 1 (b) Operation mode 2

of $\theta, \phi$ and $Z$, the altitude of the moving platform from the fixed base. The elements of the translational part $\mathbf{M}_{T}$ of matrix $\mathbf{M}$ in equation (2) are considered as $\mathbf{M}_{T}=[X, Y, Z]^{T}$ that represent the translational displacement in the coordinate axes $x, y$ and $z$ respectively. Then, the constraint equations are derived in terms of 
$X, Y, Z, x_{0}, x_{1}, x_{2}, x_{3}, r_{1}, r_{2}, r_{3}$. From these equations, the variables $X, Y, r_{1}, r_{2}$ and $r_{3}$ are expressed as a function of $Z$ and $x_{i}$ and are substituted in the determinant of the Jacobian. Finally, the corresponding $x_{i}$ are expressed in terms of Euler angles, which yields a single equation describing the actuation singularity of the 3-SPR PM in terms of $Z, \theta$ and $\phi$. Fixing the value of $Z$ and plotting the determinant of the Jacobian for $\phi \in\left[-180^{\circ}, 180^{\circ}\right]$ and $\theta \in\left[0^{0}, 180^{\circ}\right]$ depicts the singularity loci. The green curves in figure 3 (a) and 3(b) show the singularity loci for operation mode 1 and operation mode 2 respectively with $h_{1}=1, h_{2}=2$ and $Z=1$.

\section{Maximum Inscribed Circle Radius for 3-RPS and 3-PRS PMs}

From the home position of the manipulator $(\theta=\phi=0)$, a circle is drawn that has the maximum tilt value for any azimuth within the singularity-free region [7]. The radius of this circle is called the Maximum Inscribed Circle Radius (MICR). In Figure 3, the red circle denotes the maximum inscribed circle where the value of MICR is expressed in degrees.

The MICR is used as a basis to compare the 3-SPR and the 3-RPS parallel manipulators as they are analogous to each other in aspects like number of operation modes and direct kinematics. $\frac{Z}{h_{1}}$ vs MICR is plotted for different ratios of $h_{2}: h_{1}$ in Figures 4 and 5. The maximum value of MICR is limited to 160 degrees for all the figures and $\frac{Z}{h_{1}}$ varies from 0 to 4 while eight ratios of $h_{2}: h_{1}$ are considered. The data cursor in Figures 5(a) and 5(b) correspond to the red circles with MICR = 25.22 and 30.38 degrees in Figures 3(a) and 3(b), respectively. The MICR plots give useful information on the design choice of 3-RPS or 3-SPR parallel manipulators. The 3-SPR PM has higher MICR values and hence larger singularity free regions compared to that of the 3-RPS PM in compliance with [3, 4]. For 3-RPS parallel manipulator, there exists rarely any difference in the MICR values for different operation modes whereas in 3-SPR PM, the second operation mode has higher values of MICR compared to operation mode 1 . The values of MICR ranges from $0^{0}$ to $130^{\circ}$ in operation mode 1 , but from $0^{0}$ to $160^{\circ}$ in operation mode 2 for 3 -SPR PM. In addition, for 3-RPS PM, the ratio $h_{1}: h_{2}$ influences operation mode 1 more than operation mode 2 . It is apparent that the MICR values have a smaller range for different ratios in case of operation mode 2. On the contrary, for 3-SPR PM, high MICR values can be seen for operation mode 2 , for lower ratios of $h_{1}: h_{2}$. Therefore, the MICR plots can be exploited in choosing the ratio of the platform to the base in accordance with the required application. 


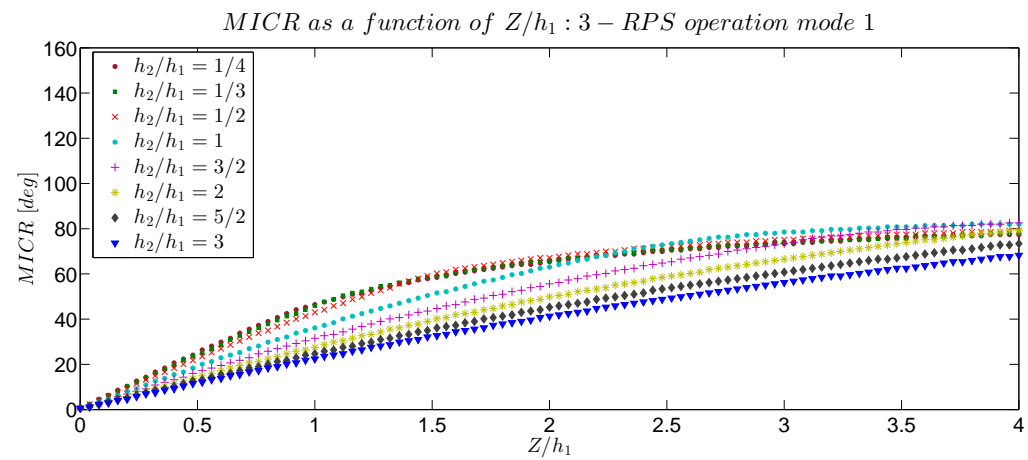

(a)

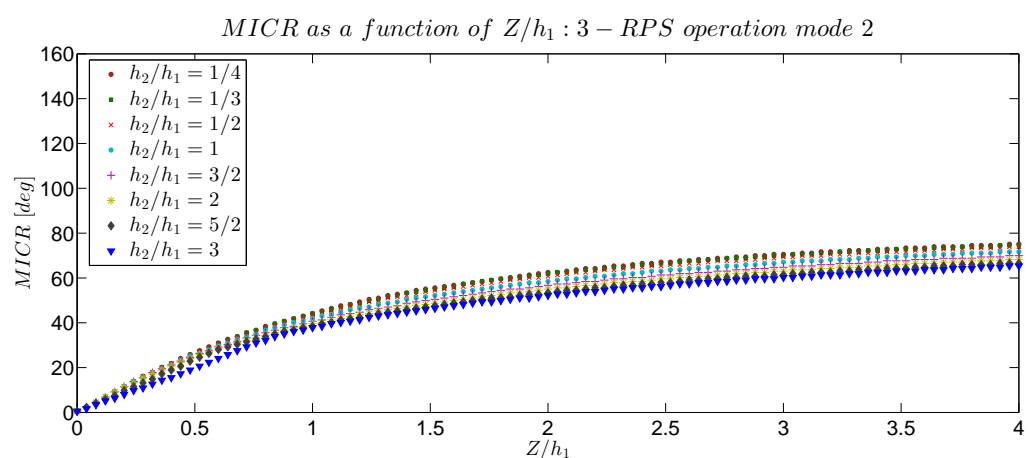

(b)

Fig. 4 MICR vs. $\frac{Z}{h_{1}}$ for the 3-RPS manipulator (a) Operation mode 1 (b) Operation mode 2

\section{Conclusions}

In this paper, 3-RPS and 3-SPR parallel manipulators were compared based on their operation modes and singularity-free workspace. Initially, the operation modes of the 3-SPR PM were enumerated. It turns out that the 3-SPR parallel manipulator has two operation modes similar to the 3-RPS PM. The parallel singularities were computed for both the manipulators and the singularity loci were plotted in their orientation workspace. Furthermore, an index called the singularity-free maximum inscribed circle radius was defined. MICR was plotted as a function of the $Z$ coordinate of the moving-platform for different ratios of the platform circum-radius to the base circum-radius. It shows that, compared to the 3-RPS PM, the 3-SPR PM has higher MICR values and hence a larger singularity free workspace for a given altitude. For the ratios of the platform to base size, higher values of MICR are observed in operation mode 2 than in operation mode 1 for the 3-SPR mechanism and is viceversa for the 3-RPS mechanism. In fact, the singularity-free MICR curves open up many design possibilities for both mechanisms suited for a particular application. 


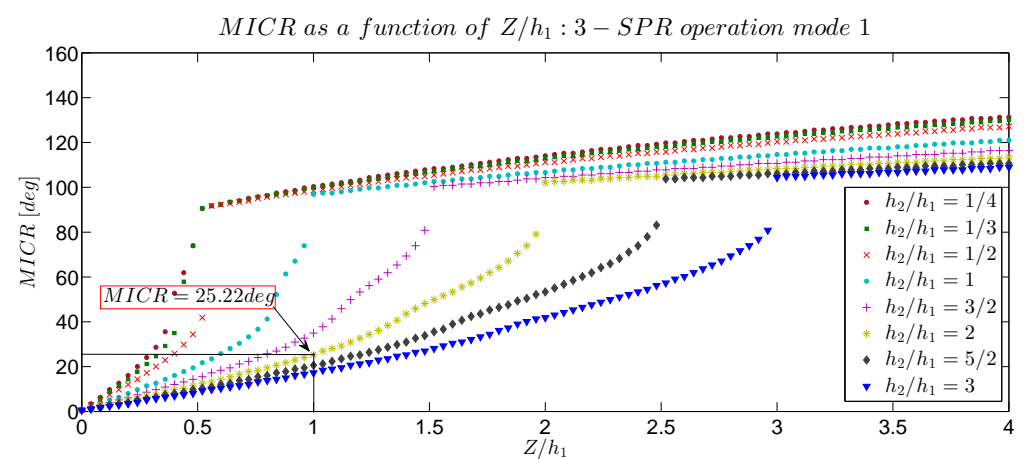

(a)

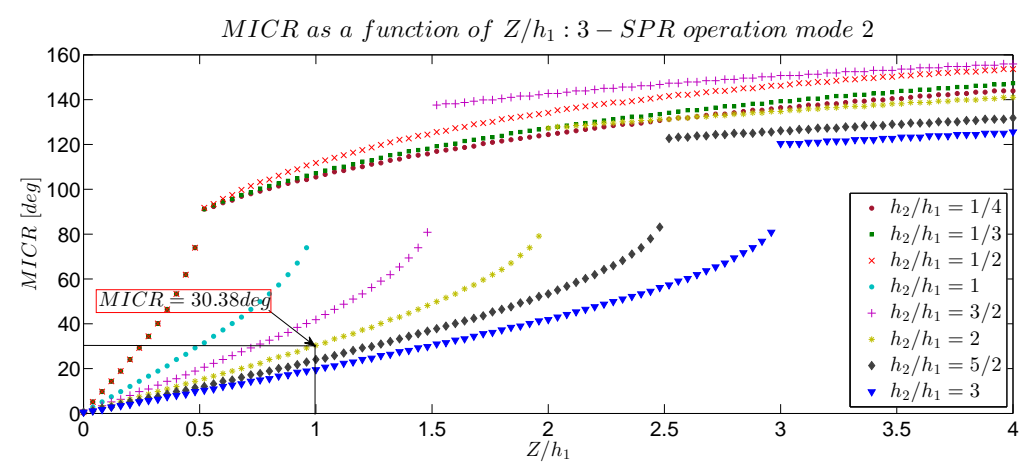

(b)

Fig. 5 MICR vs. $\frac{Z}{h_{1}}$ for the 3-S $\underline{P R}$ manipulator (a) Operation mode 1 (b) Operation mode 2

It will also be interesting to plot the MICR curves for constraint singularities and other actuation modes like 3-표 and 3-SPR manipulators and to consider the parasitic motions of the moving-platform within the maximum inscribed circles. The investigation of MICR not started from the identity condition $(\theta=\phi=0$ degrees $)$ has to be considered too. Future work will deal with those issues.

\section{References}

1. Josef Schadlbauer, Dominic R. Walter and Manfred L. Husty : A Complete Kinematic Analysis of the 3-RPS Parallel Manipulator, 15th National Conference on Machines and Mechanisms (2011).

2. Schadlbauer J., Walter D.R., and Husty M.: The 3-RPS Parallel Manipulator from an Algebraic Viewpoint, Mechanism and Machine Theory, 75, pp. 161-176 (2014).

3. V. Lukanin, Inverse Kinematics, Forward Kinematics and Working Space Determination of 3DOF Parallel Manipulator with S-P-R Joint Structure, Periodica Polytechnica Ser. Mech.Eng. Vol.49, No.1, pp.39-61 (2005). 
4. Y. Lu and Y. Zhao, Position and Workspace Analysis of 3-SPR and 3-RPS Parallel Manipulators, Proceedings of IDETC/CIE, September (2005).

5. Mark W., Martin W., Stefan N., Christoph G., Werner S. and Oliver S.:Kinematic Modeling of a Hydraulically Actuated 3-SPR-Parallel Manipulator for an Adaptive Shell Structure, ASME (AIM), Wollongong, Australia, July 9-12 (2013).

6. Nurahmi L., Caro S. , Wenger P., : Operation modes and singularities of 3-PRS parallel manipulators with different arrangements if P-joints, IDETC/CIE, Boston, Massachusetts, USA (2015).

7. Sébastien Briot and Ilian A. Bonev : Singularity Analysis of Zero-Torsion Parallel Mechanisms, International Conference on Intelligent Robots and Systems, Nice, France, Sept 22-26 (2008).

8. David Cox, John Little, Donald O'Shea: Ideals, Varieties, and Algorithms (Series: An Introduction to Computational Algebraic Geometry and Commutative Algebra). Springer (2006).

9. Xianwen Kong: Reconfiguration analysis of a 3-DOF parallel mechanism using Euler parameter quaternions and algebraic geometry method, Mechanism and Machine Theory, 75, pp. 188-201 (2014)

10. Schadlbauer J., Nurahmi L., Husty, M., Wenger P., Caro. S : Operation Modes in LowerMobility Parallel Manipulators, in Second Conference on Interdisciplinary Applications of Kinematics, Lima, Peru, pp. 3-10 (2013). NASA Mission Planning and Analysis Division, Euler Angles, Quaternions, and Transformation Matrices, London B Johnson Space Center Houston Texas (1977). 